\title{
Aggregation and gelation in hydroxypropylmethyl cellulose aqueous solutions
}

\author{
Sérgio M.C. Silva ${ }^{\mathrm{a}, \mathrm{b}}$, Fátima V. Pinto ${ }^{\mathrm{a}}$, Filipe E. Antunes ${ }^{\mathrm{c}}$, Maria G. Miguel ${ }^{\mathrm{a}}$, João J.S. Sousa ${ }^{\mathrm{b}}$, Alberto A.C.C. Pais ${ }^{\mathrm{a}, *}$ \\ a Chemistry Department, University of Coimbra, Rua Larga, 3004-535 Coimbra, Portugal \\ ${ }^{\mathrm{b}}$ Faculty of Pharmacy, University of Coimbra, Rua do Norte, 3000-295 Coimbra, Portugal \\ c Chemical Engineering Department, University of Coimbra, Polo II - Pinhal de Marrocos, 3030-290 Coimbra, Portugal
}

\section{A R T I C L E I N F O}

\section{Article history:}

Received 17 July 2008

Accepted 28 August 2008

Available online 4 September 2008

\section{Keywords:}

Hydroxypropylmethyl cellulose

Gelation mechanism

Rheology

Micropolarity index

\begin{abstract}
A B S T R A C T
In this work we present an analysis of the thermal behavior of hydroxypropylmethyl cellulose aqueous solutions, from room temperature to higher temperatures, above gelation. We focus on significant aspects, essentially overlooked in previous work, such as the correlation between polymer hydrophobicity and rheological behavior, and the shear effect on thermal gelation. Micropolarity and aggregation of the polymer chains were monitored by both UV/vis and fluorescence spectroscopic techniques, along with polarized light microscopy. Gel formation upon heating was investigated using rheological experiments, with both large strain (rotational) tests at different shear rates and small strain (oscillatory) tests. The present observations allow us to compose a picture of the evolution of the system upon heating: firstly, polymer reptation increases due to thermal motion, which leads to a weaker network. Secondly, above $55^{\circ} \mathrm{C}$, the polymer chains become more hydrophobic and polymer clusters start to form. Finally, the number of physical crosslinks between polymer clusters and the respective lifetimes increase and a three-dimensional network is formed. This network is drastically affected if higher shear rates, at nonNewtonian regimes, are applied to the system.
\end{abstract}

(C) 2008 Elsevier Inc. All rights reserved.

\section{Introduction}

Water soluble cellulose derivatives are mostly biocompatible polymers used in a wide range of applications, especially in food, pharmaceutical, and cosmetic industries [1,2]. In particular, when dissolved in water, they can be used as thickeners, binding agents, emulsifiers, film formers, suspension aids, surfactants, lubricants and stabilizers [3]. The most common cellulose derivatives include methyl cellulose, hydroxypropyl cellulose, carboxymethyl cellulose and hydroxypropylmethyl cellulose (HPMC). Most of the applications of these cellulose derivatives solutions involve heating the polymer, and so it is crucial to understand the temperature dependence behavior of these solutions, which has been the object of various studies [4].

Cellulose is a natural polysaccharide, structurally consisting of a linear homopolymer backbone formed by D-glucopyranose units linked by $\beta 1-4$ glycosidic bonds [5,6]. Pure cellulose (Fig. 1a) is insoluble in water due to the existence of strong intra-molecular interactions via hydrogen bonds, which represents a limitation in practical applications. This can be overcome by preparing water soluble cellulose derivatives. The derivatives result from the substitution of hydroxyl groups in each anhydroglucose ring by

\footnotetext{
* Corresponding author. Fax: +351 239827703.

E-mail address: pais@qui.uc.pt (A.A.C.C. Pais).
}

other functional groups that decrease the crystallinity of the substance [7].

In the case of HPMC, the hydroxyl groups are replaced by hydroxypropyl and methyl groups as seen in Fig. 1b. There are three types of HPMC classified by the United States Pharmacopea according to the chemical substitution of the ether: E (hypromellose 2910), F (hypromellose 2906) and $\mathrm{K}$ (hypromellose 2208). The major chemical differences between these types of HPMC are in the degree of substitution, molar degree of substitution and degree of polymerization. The degree of substitution corresponds to the average number of substituted hydroxyl groups (maximum of 3 ), and the molar degree of substitution gives the number of substituents introduced into the anhydroglucose unit. Finally, the degree of polymerization is related to the average number of monomers in the chains [3].

HPMC aqueous solutions generally have the property of forming a reversible gel with temperature. Nevertheless, the study and understanding of this process is complex due the possibility of several different phenomena occurring during the heating cycle [810]. It is generally accepted that the thermoreversible gelation of HPMC solutions is due to hydrophobic interactions, and it is sometimes indicated that clouding precedes gelation $[11,12]$.

Gelation can be detected by a sharp increase in viscosity [13]. The temperature at which this occurs is influenced by the type of cellulose ether (see e.g. Ref. [4]). It has been observed that methyl- 


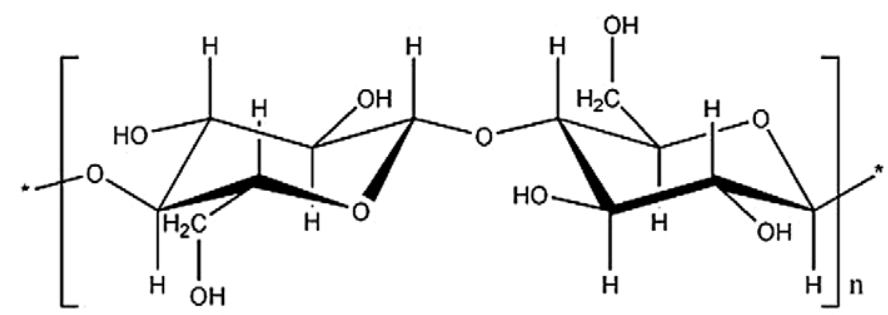

(a)

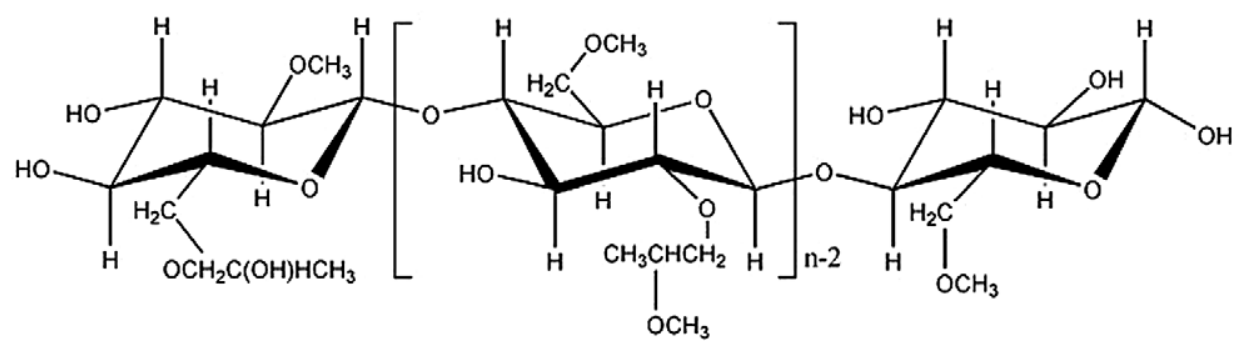

(b)

Fig. 1. Structure of (a) natural cellulose, and (b) hydroxypropylmethyl cellulose (HPMC).

cellulose has a lower gelation temperature and forms firmer gels than HPMC with equivalent substitution and molecular weight, suggesting that hydroxypropyl substituents make the gelation process more difficult [14,15]. Additionally, hydroxypropyl cellulose precipitates with increasing temperature but does not form a gel, which has been seen as evidence that the gelation of cellulose derivatives results from the exclusion of water (syneresis) from heavily methoxylated regions of the polymer $[12,16]$. Conformational changes similar to those occurring in polyethyleneoxide have been presented as an explanation for the increasing hydrophobicity as temperature increases in substituted celluloses [17]. It is generally accepted that a polymer that provides such properties usually carries two different segments: one hydrophobic and one hydrophilic, distributed along the polymer chain. However, at higher temperatures the influence of the hydrophobic group is predominant [18]. Some authors also suggest that not only hydrophobic interaction but also hydrogen bonding may be involved in the gelation mechanism of cellulose derivatives [19].

The gelation phenomenon can be studied by a variety of techniques, which in turn provide different observables and different definitions for the gelation temperature. In this work, we use rheology to study the thermal behavior of a range of HPMC solutions. In particular, we investigate various factors that can be used to establish the gelation temperature. These include the "classical approach" in which a ramp is used to set an increasing temperature, where gelation is monitored through a sudden alteration in viscosity. We also look into how the viscosity varies (and gelation temperature) for different shear rates. Issues related to kinetic effects on gelation are discussed in conjunction with this approach. Oscillatory measurements give additional information on the system, and provide another estimate of the gelation temperature. UV/vis spectroscopy measurements are used to determine the cloud point $(\mathrm{CP})$. This technique, together with polarized light thermal microscopy (PLTM) is important to assess the formation and growth of polymer aggregates that will ultimately lead to a three-dimensional gel network.

In this study, fluorescence spectroscopy using pyrene as a probe, is performed in order to observe how the hydrophobic character of the polymer domains varies with temperature.

\section{Experimental}

\subsection{Materials and sample preparation}

The cellulose derivative HPMC (Methocel ${ }^{\circledR}$ K15M Premium), 19$24 \%$ methoxyl and 7-12\% hydroxypropyl, $M_{w}=4.3 \times 10^{5}$ Da was purchased from Dow Chemical Company and was used without further purification. Aqueous solutions of HPMC of different concentrations $(1,2,5$ and $10 \%, \mathrm{w} / \mathrm{w}$ ) were prepared by adding the desired amount of dry polymer to Millipore ${ }^{\circledR}$ water. The polymer solutions are above overlap concentration. Due to the long dissolution times, solutions were kept stirring for one week before use.

\subsection{Rheological studies}

The rheological measurements were conducted on a Reologica ${ }^{\circledR}$ Stresstech stress controlled rheometer equipped with automatic gap setting. A bob cup measurement system (CC 15) with a solvent trap was used to test all samples. The temperature control was achieved using a water bath system. All experiments based on a temperature ramp were performed from 25 to $90^{\circ} \mathrm{C}$ at a fixed heating rate of $1^{\circ} \mathrm{C} / \mathrm{min}$. A number of viscosity measurements were also conducted in steady-state conditions. Dynamic or shear viscosity was analyzed by rotational shear experiments. Prior to this, a viscometry test was carried out in order to determine both Newtonian and non-Newtonian regimes. Oscillation tests were performed to determine the storage modulus, $G^{\prime}$, and loss modulus, $G^{\prime \prime}$. Oscillatory measurements can be used to study the structure of a material, in which the deformation in the oscillation has to be kept small. An important point is to check whether measurements are made in the linear viscoelastic regime, otherwise the results of the frequency experiments will depend not only on the frequency but also on the applied stress or deformation. A test of linearity, the oscillation stress sweep, was used to obtain the values of shear stress for which the viscoelastic functions are independent from the magnitude of the applied stress. The applied frequency was chosen ensuring that, at the initial conditions, $G^{\prime \prime}$ is dominant. The complex viscosity modulus $\eta^{*}$, a mathematical representation of the viscosity in oscillatory tests [20], was also obtained. Finally, relaxation times were extracted from the moduli $G^{\prime}$ and $G^{\prime \prime}$, represented as a function of the frequency (frequency sweep tests). 


\subsection{Cloud point determination and polarized light thermal microscopy observations}

An ultraviolet-visible spectrophotometer (Shimadzu UV-2100) was used for the cloud point measurements. The sample was placed in a cell with a dimension of $1 \times 1 \times 5 \mathrm{~cm}$. Millipore ${ }^{\circledR}$ water was used as reference. For temperature measurements, the cells were placed in a cell-holder connected to a water bath system with temperature regulation (Julabo F30-C). In addition to this regulator, an external temperature sensor ( $\mathrm{Pt} 100$ ) was placed in the sample cell. The absorption spectra (200-850 nm) confirmed that there were no UV-absorbing moieties in the solutions. The transmittance was measured at a wavelength of $800 \mathrm{~nm}$ as previously reported for a similar system [11]. The system was heated at a faster rate at lower temperatures. This was then reduced to a slower heating rate at the point where the transmittance started to decrease. The system was equilibrated for at least $30 \mathrm{~min}$ before each measurement.

The cloud point has been related to gelation, and different definitions were used to determine this point. In theory, the cloud point is defined as the temperature at which phase separation, in the form of turbidity, occurs for a $1 \%$ polymer concentration [21]. However, in practical applications the cloud point can be defined as the intersection of two straight lines drawn through the curves of absorbance at low and high temperatures, respectively [22], or as the temperature corresponding to $50 \%$ of the transmittance found for some reference value $[11,23]$ or as the maximum in the first derivative of absorbance in relation to temperature [24]. In this study the cloud point was considered to be the temperature at which the light transmittance was $50 \%$ of that obtained for the same sample at $25^{\circ} \mathrm{C}$.

The microscopy analysis was performed using a Linkam system DSC 600. The optical observations were conducted using a LeicaDMRB microscope and registered using a Sony CCD-IRIS/RGB video camera. The image analysis used a Linkam system software with the Real Time Video Measurement System. The images were obtained combining the use of polarized light with wave compensators, at a $200 \times$ magnification. The thermal behavior of the solutions was studied in heating/cooling cycles between 25 and $90^{\circ} \mathrm{C}$, at scanning rates of $5^{\circ} \mathrm{C} / \mathrm{min}$. The scans were made under a nitrogen atmosphere through the use of nitrogen flow.

\subsection{Pyrene fluorescence measurements}

The ratio of the first $\left(I_{1}, \lambda=374 \mathrm{~nm}\right)$ and third $\left(I_{3}, \lambda=\right.$ $388 \mathrm{~nm})$ vibronic fluorescence emission intensities $\left(I_{1} / I_{3}\right)$ of pyrene is known as the micropolarity index, and is a qualitative measurement of the polarity at the local environment of the probe [25]. This index is close to 2.0 in pure water and ca. 1.0 in hydrophobic solvents like toluene.

Being a hydrophobic probe, pyrene presents low solubility in water (approximately $10^{-6} \mathrm{M}$ ). Pyrene solutions were prepared by filtering pyrene-satured Millipore ${ }^{\circledR}$ water. The samples were prepared in situ by adding $30 \mu \mathrm{L}$ of the before mentioned solution to $3 \mathrm{~mL}$ of $1 \%$ HPMC K15M solution and were left to equilibrate under stirring for $15 \mathrm{~min}$ inside of the measurement cell.

The fluorescence spectra were recorded with a Horiba-JobinIvon SPEX Fluorolog 3-22 spectrometer and were corrected for the instrumental response. The Fluorolog consists of a modular spectrofluorimeter with a double grating excitation (range 200$950 \mathrm{~nm}$, optimized in the UV and with a blazed angle at $330 \mathrm{~nm}$ ) and emission (range 200-950 $\mathrm{nm}$, optimized in the visible and with a blazed angle at $500 \mathrm{~nm}$ ) monochromators. The bandpass for excitation and emission is $0-15 \mathrm{~nm}$ (values that are continuously adjustable from computer Datamax/32 software) and the wavelength accuracy is of $0.5 \mathrm{~nm}$. The excitation source consists in an ozone-free $450 \mathrm{~W}$ xenon lamp and the emission detector is a Hamamatsu R928 Photomultiplier (200-900 nm range), cooled with a Products for Research thermoelectric refrigerated chamber (model PC177CE005) and a photodiode as the reference detector.

We note the system was equilibrated for at least $30 \mathrm{~min}$ before the measurement at each temperature.

\section{Results}

In this work we present four different strategies to investigate the thermal behavior of HPMC solutions: (i) the dependence of viscosity on temperature, at different shear rates through rotational tests; [26] (ii) the influence of temperature on the storage and loss moduli by oscillatory measurements, with inspection of relaxation times; (iii) clouding studies carried out via optical transmittance and PLTM measurements, and (iv) the evolution of the system polarity through fluorescence spectroscopy using pyrene as a probe.

\subsection{Rotational measurements at different shear rates}

Fig. 2 shows the viscosity-temperature behavior of the polymer solutions for the different concentrations of HPMC. For each solution, we have studied the viscosity upon heating for different values of the shear rate. Let us first address the results at the lower, Newtonian, shear rates. As the temperature rises, the viscosity smoothly decreases up to a characteristic temperature, usually denoted as the gelation temperature [11]. A sharp increase in viscosity is seen after this temperature. This characteristic point is concentration dependent, with a tendency for a decrease with concentration from $2 \%$ onwards. For each temperature point the viscosity increases with HPMC content, as expected.

For the higher shear rates, corresponding to non-Newtonian regimes, viscosity suddenly drops close to the temperature at which gelation occurs for Newtonian shear rates. Then, this quantity reaches its lowest value and forms a plateau. The plateaus for higher and lower shear rates are observed for similar values of the temperature.

Viscosity vs. temperature plots for the $2 \%$ solution were also obtained with a systematic variation in the shear rate (see Fig. 3). It is observed that, with increasing shear rate, the point at which viscosity starts to increase is moved towards higher temperatures. In our observations, shear rates of 8,10 and $30 \mathrm{~s}^{-1}$ tend to prevent gelation, replacing the steep increase in viscosity by a not well defined plateau slightly above the global minimum.

This dependence is again highlighted in Fig. 4. For lower temperatures, below gelation, it is seen that the effect of shear rate upon viscosity, in this case shear thinning, is limited. As the temperature increases the viscosity diminishes as previously shown, but the Newtonian regime remains essentially unaffected. Above $70^{\circ} \mathrm{C}$ the behavior changes dramatically, and shear thinning amounts to several orders of magnitude. Basically, low shear rates correspond to very high viscosities (gel) but, when a sufficiently large shear rate is applied, viscosity sharply decreases. Note also the general good agreement between the values of viscosity depicted in the Newtonian regimes for each temperature, and those found in Fig. 2b for the lower shear rates. This indicates an appropriate choice of the shear rates used in the studies in which a temperature ramp is imposed, even for temperatures above gelation.

An issue that is not often addressed in these systems is the kinetic control over the gelation process. Tests conducted with the $2 \%$ HPMC solution show that gelation, with a viscosity close to $200 \mathrm{Pas}$, occurs at ca. $75^{\circ} \mathrm{C}$ if the system is left to evolve at this temperature for more than one hour, so as to reach the steadystate (data not shown). At lower temperatures, we have not been able to detect gelation. 


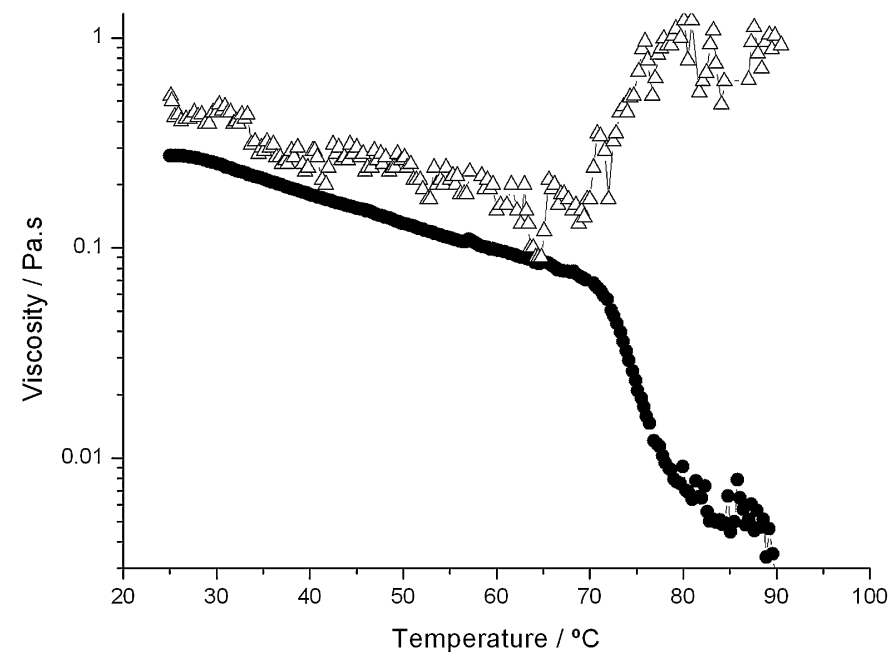

(a)

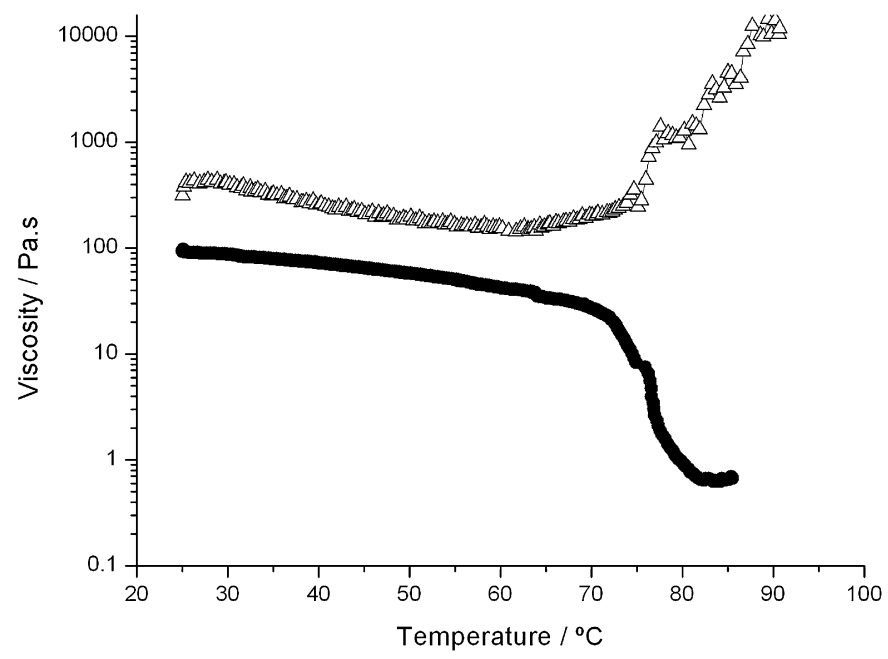

(c)

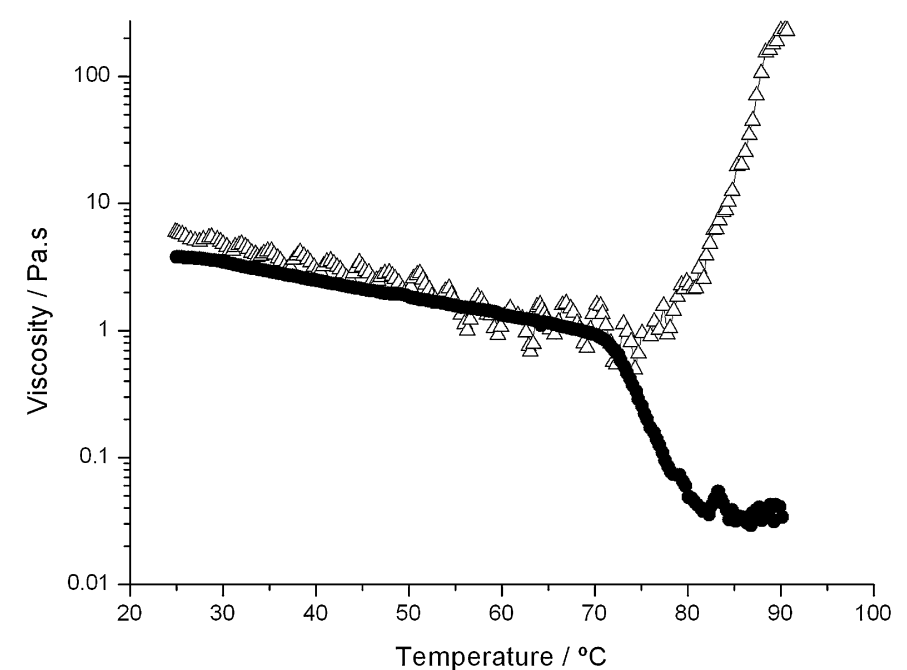

(b)

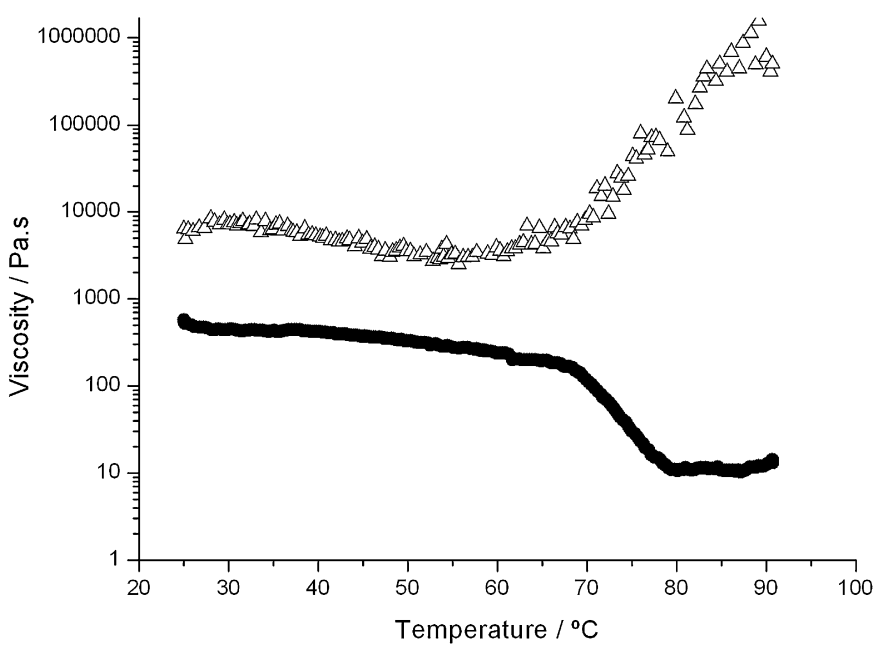

(d)

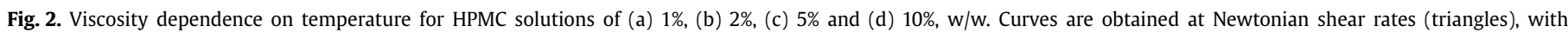
imposed values respectively of $0.5,0.05,5.0 \times 10^{-3}$ and $1.0 \times 10^{-3} \mathrm{~s}^{-1}$, and non-Newtonian shear rates (circles), of $50,30,5$ and $1 \mathrm{~s}^{-1}$ respectively.

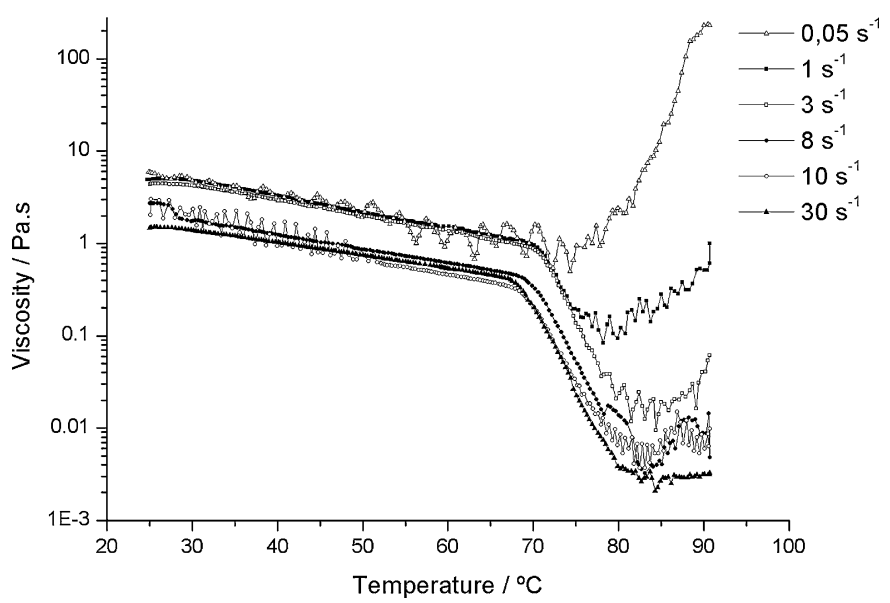

Fig. 3. Viscosity as a function of temperature for the indicated shear rates in the $2 \%$ HPMC solution.

It is thus likely that determinations such as those depicted in Fig. 2, corresponding to a rate of increase in temperature of $1{ }^{\circ} \mathrm{C} /$ min (in which evaporation effects are minimized), may reflect some delay for establishing the actual value of the viscosity. However, the gelation temperature as extracted from the increase

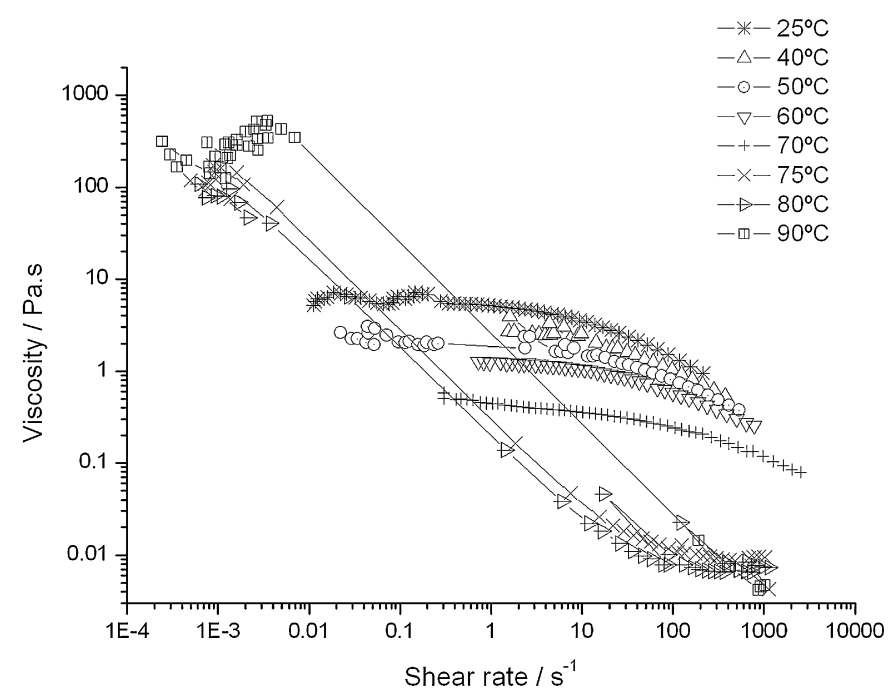

Fig. 4. Viscosity as a function of shear rate in the $2 \%$ HPMC solution at the indicated temperatures.

in viscosity is essentially accurate. It should be added that studies on the time evolution in methylcellulose systems have shown that significant equilibration occurs after ca. $5 \mathrm{~min}$ after temperature 


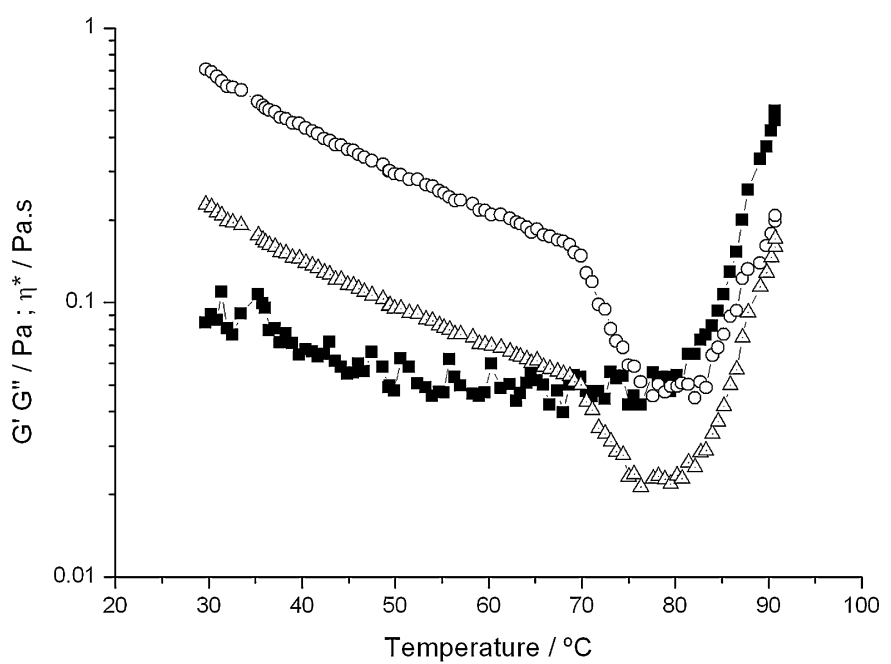

(a)

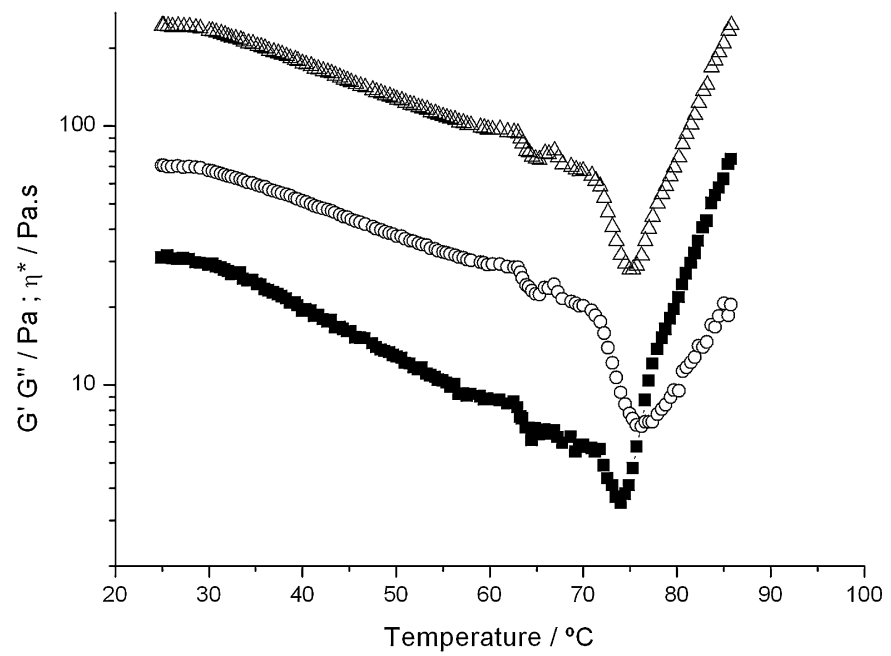

(c)

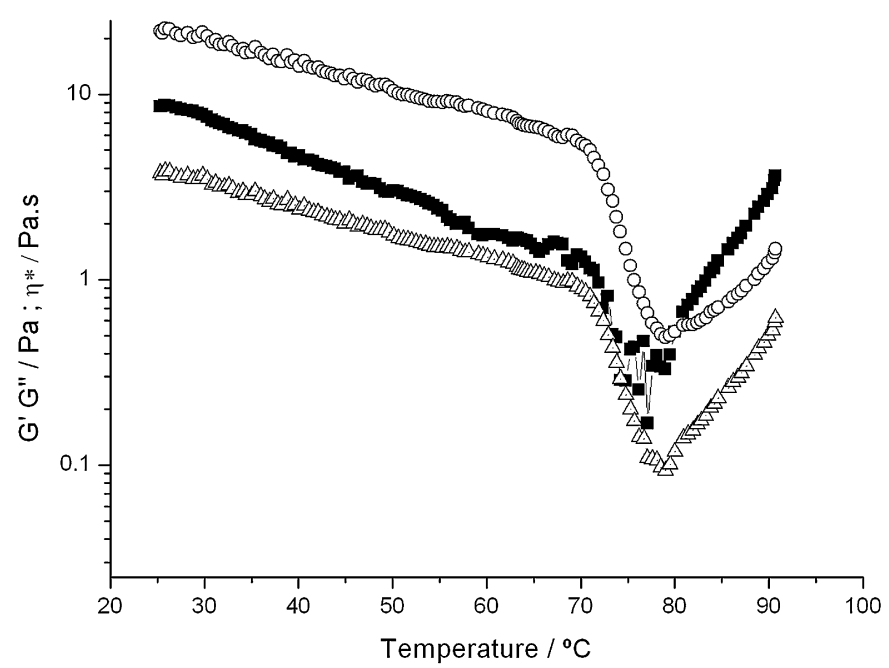

(b)

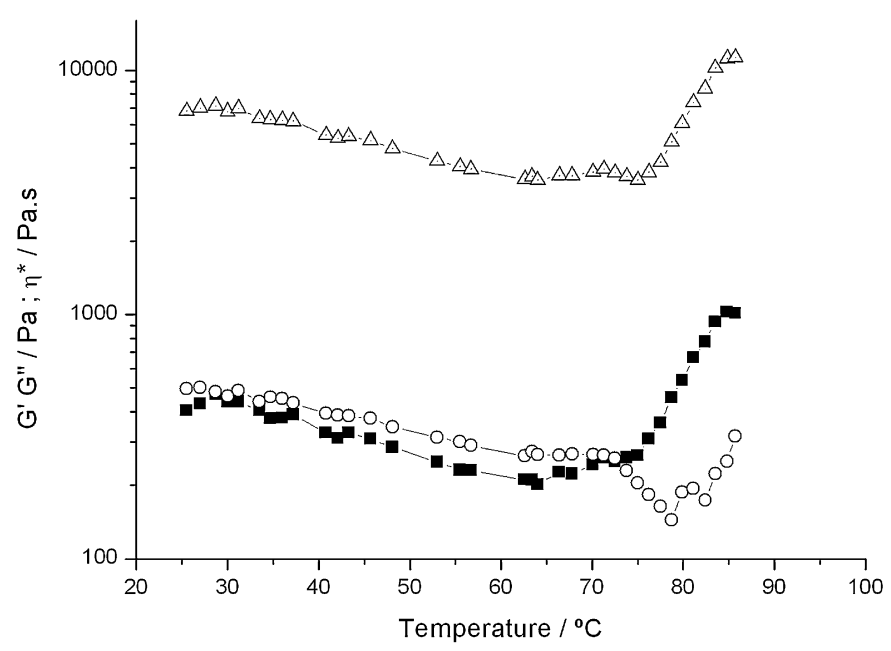

(d)

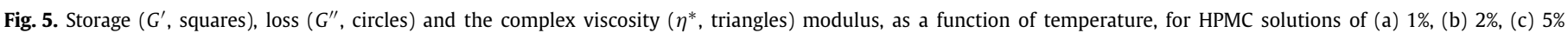
and (d) $10 \%$, w/w. Frequency is chosen so as to impose a value of $G^{\prime \prime}$ higher than $G^{\prime}$ at the initial conditions.

stabilization [9]. Thus, the studies based on light transmittance and pyrene fluorescence are performed on an essentially equilibrated system.

\subsection{Oscillatory measurements}

The temperature effect on gelation of the HPMC solutions was also studied by oscillatory tests and the results are shown in Fig. 5 . In general, as the temperature is increased, $G^{\prime \prime}$ smoothly decreases until reaching a characteristic temperature after which a sharp decrease is observed. $G^{\prime}$ shows a similar behavior, but the sharp decrease is less pronounced when compared to $G^{\prime \prime}$, and absent in the case of the $1 \%$ solution. It is interesting to note that this characteristic temperature is observed slightly above $70^{\circ} \mathrm{C}$ for all concentrations of this study, being independent of the concentration of HPMC. Also observed is a final increase in both moduli with temperature, which is more significant for the storage modulus than for the loss modulus, at which $G^{\prime}$ and $G^{\prime \prime}$ curves intersect. At this point, the solution starts to gel as can be noticed by the sharp increase in the complex viscosity modulus. This is considered by a number of authors to be the gelation temperature [27, 28]. However, this crossing point depends both on the concentration of the polymer and on the frequency applied on oscillatory tests. It should also be noted that $G^{\prime}$ values at the end of the tests are always higher than at the initial conditions $\left(25^{\circ} \mathrm{C}\right)$, but
Table 1

Relaxation times, calculated by the intersection of $G^{\prime}$ and $G^{\prime \prime}$ in the frequency sweep tests at constant temperature, and storage modulus $G^{\prime}$ at $1 \mathrm{~Hz}$. Data pertain to the $2 \%$ HPMC solution, and samples were in the steady-state

\begin{tabular}{lcl}
\hline Temperature $\left({ }^{\circ} \mathrm{C}\right)$ & Relaxation time $(\mathrm{s})$ & $G^{\prime}(\mathrm{Pa})$ \\
\hline 40 & 0.058 & 4.24 \\
60 & 0.106 & 2.94 \\
85 & 15.9 & 4.68 \\
\hline
\end{tabular}

the same is not true for the $G^{\prime \prime}$ measurements. It is seen that the absolute values of the moduli are higher with increasing HPMC content.

Relaxation times, given by the intersection of $G^{\prime}$ and $G^{\prime \prime}$ in the frequency sweep tests at constant temperature, and storage moduli at $1 \mathrm{~Hz}$, with long equilibration times, are shown in Table 1 for the $2 \%$ solution. It is seen that, while the relaxation time always increases with temperature, the storage modulus $G^{\prime}$ shows a nonmonotonic behavior.

\subsection{Optical transmittance measurements and polarized light thermal microscopy}

The light transmittance of HPMC solutions at the wavelength of $800 \mathrm{~nm}$ is plotted in Fig. 6 as a function of temperature. The 


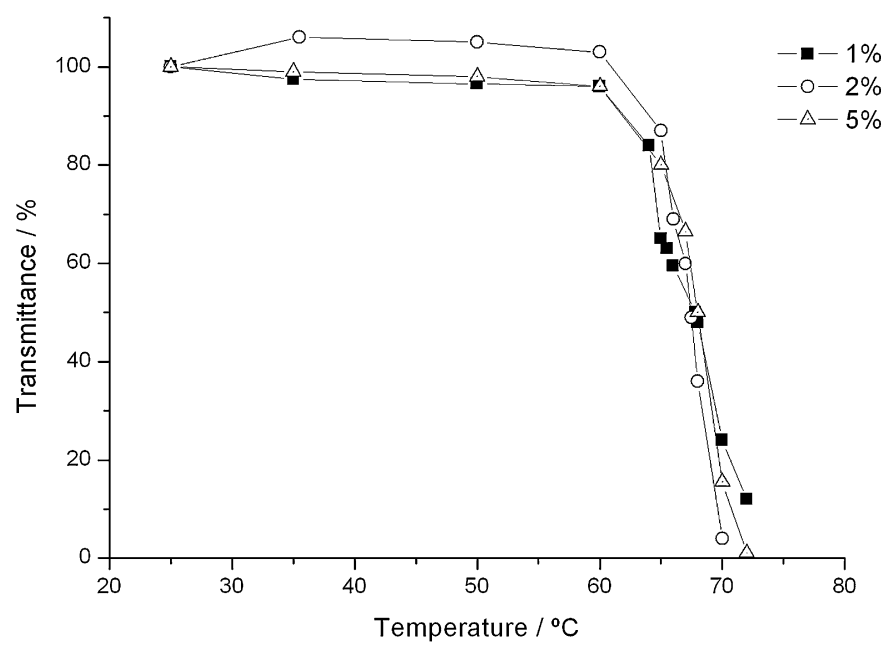

Fig. 6. Optical transmittance measurements for the HPMC solution for the indicated concentrations. The $100 \%$ reference was taken as the transmittance at $25^{\circ} \mathrm{C}$ for each solution.

highest concentration requires very high equilibration times, and was excluded. For HPMC concentrations between 1 and $5 \%$ it is seen that the transmittance curves have a very similar temperature profile. A long plateau in which transmittance is hardly affected ( $100 \%$ relative to the value at $25^{\circ} \mathrm{C}$ ) is followed by a sharp decrease at ca. $60^{\circ} \mathrm{C}$, which reaches $0 \%$ at about $70^{\circ} \mathrm{C}$. The cloud point, i.e. the reduction to $50 \%$ of the value at $25^{\circ} \mathrm{C}$, is observed at ca. $68^{\circ} \mathrm{C}$ for every concentration.

Observations from PLTM confirm an isotropic liquid at lower temperatures as shown in Fig. 7a, characteristic of the polymer sol. This behavior is seen up to temperatures close to gelation. As the temperature is increased above this point, the system exhibits some texture and, at the same time, light transmission is reduced as observed in Fig. $7 \mathrm{~b}$.

\subsection{Pyrene fluorescence measurements}

The results on the evolution of the $I_{1} / I_{3}$ ratio are shown in Fig. 8. We recall that a value slightly below 2 is expected for aqueous pyrene solutions, while values close to 1 are attained in apolar solvents. The $1 \%$ HPMC solution possesses some hydrophobic character, which surpasses that of methylcellulose [29], probably due to the presence of the hydroxypropyl substituents. The value of $I_{1} / I_{3}$ does not significantly change until ca. $55^{\circ} \mathrm{C}$. When the temperature is further increased, the emission intensity ratio decreases abruptly, after which the decrease becomes less pronounced (from $75^{\circ} \mathrm{C}$ onwards). This result is compatible with the optical light transmission measurements and PLTM observations.

\section{Discussion}

In what follows we will address different aspects of the thermal behavior of HPMC aqueous solutions. For a more systematic approach, these are divided in several relevant topics.

\subsection{Aggregation and gelation}

From the results, we can infer that gelation and aggregate formation upon heating are correlated. Between 25 and $55^{\circ} \mathrm{C}$, the fluorescence observations suggest that the character of the hydrophobic domains remains unchanged, and thus no significant aggregation of the polymer chains is expected. Within this temperature range, rheological data show a decrease in viscosity upon heating, following closely an Arrhenius behavior, with activation energies

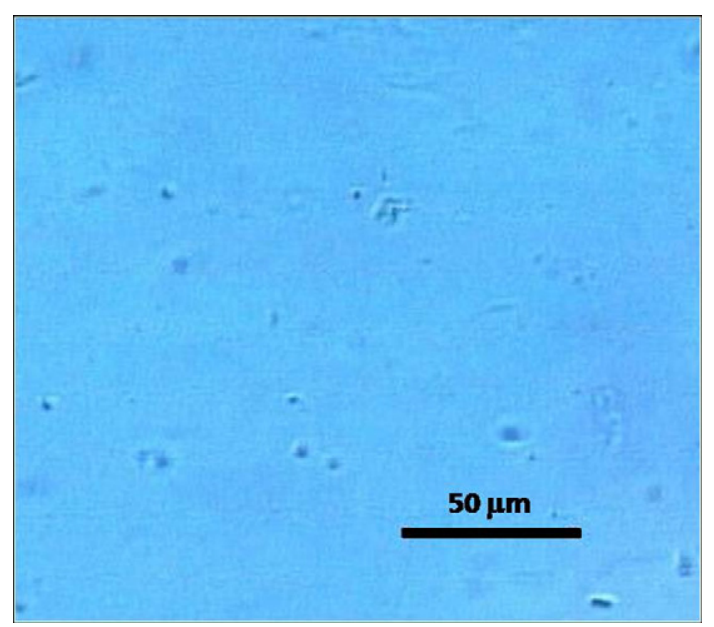

(a)

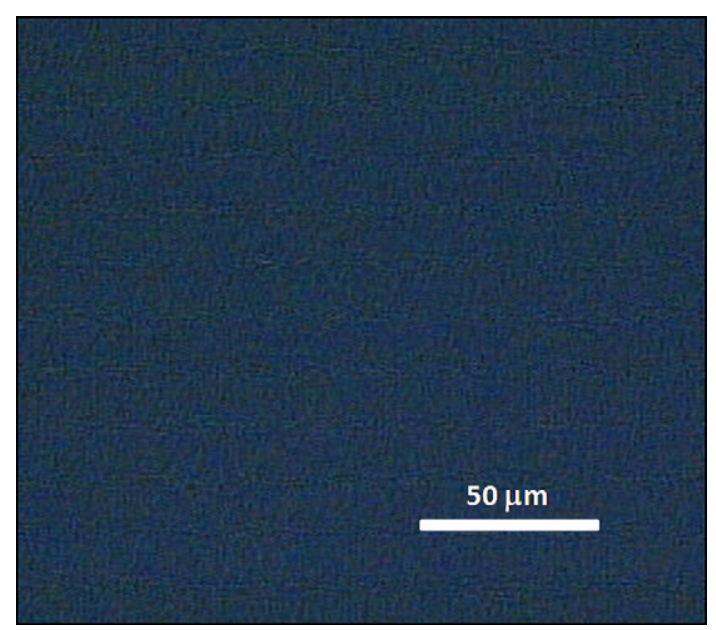

(b)

Fig. 7. PLTM images for the $1 \%$ HPMC solution for the indicated temperatures (a) $25^{\circ} \mathrm{C}$ and (b) $90^{\circ} \mathrm{C}$.

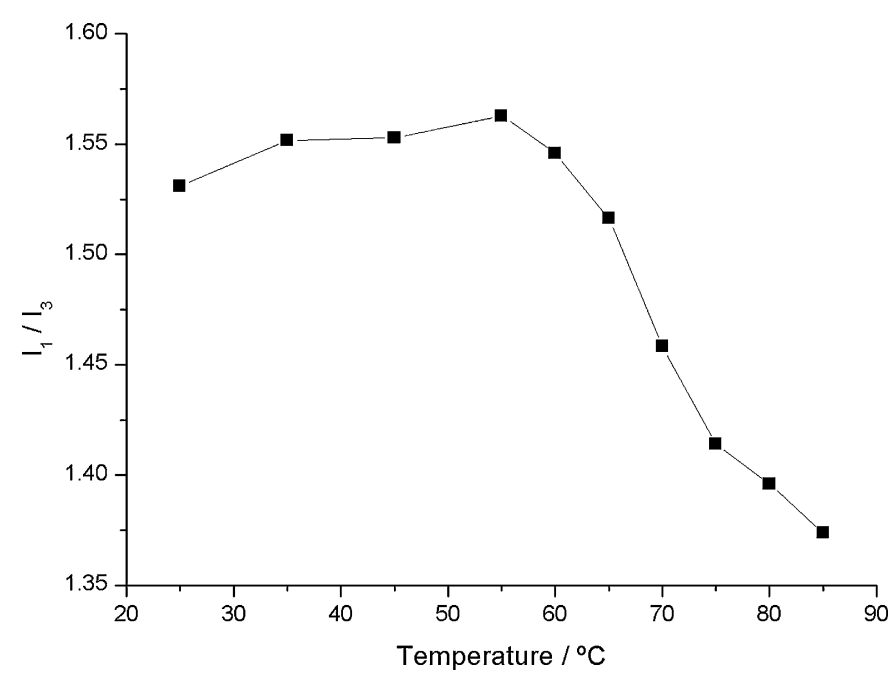

Fig. 8. $I_{1} / I_{3}$ ratio of pyrene emission or micropolarity index (MI) progression with temperature for the $1 \%$ HPMC solution.

within $22-30 \mathrm{~kJ} \mathrm{~mol}^{-1}$ for the solutions under study. This is probably due to the weakening of intermolecular interactions because of the higher average speed of molecules and of the shorter times they spend in contact with the nearest neighbors.

However, from $55^{\circ} \mathrm{C}$ onwards, fluorescence results show an increase in the hydrophobic character of the system that is respon- 
Table 2

Characteristic temperatures, in ${ }^{\circ} \mathrm{C}$, for HPMC solutions of the indicated concentrations

\begin{tabular}{cllll}
\hline \% HPMC & Cloud point & $T_{c 1}$ & $T_{c 2}$ & $T_{c 3}$ \\
\hline 1 & 68 & 70 & 71 & 78 \\
2 & 68 & 75 & 71 & 80 \\
5 & 68 & 73 & 71 & 76 \\
10 & - & 70 & 71 & 72 \\
\hline
\end{tabular}

The cloud point is described in Section 2.3. $T_{c 1}$ corresponds to the minimum before the sharp increase in viscosity at Newtonian shear rates and $T_{c 2}$ to the steep lowering in viscosity at high shear rate. Finally, $T_{c 3}$ is given by the intersection between the storage, $G^{\prime}$, and loss, $G^{\prime \prime}$, moduli.

sible for the polymer aggregation detected in the optical transmittance and PLTM studies. At a later stage, these aggregates are interconnected forming a three-dimensional network structure, and the shear viscosity increases. The variation in the hydrophobic character is relatively abrupt, contrasting to what happens in the case of methylcellulose [30]. Our rheological results indicate a delay between the onset of gelation, irrespective of the observable used for its definition, and full gelation, corresponding to the maximum in viscosity. The same picture was found by other authors using heat capacity at constant pressure to extract the gelation temperature in HPMC solutions [31]. These authors establish a degree of gelation, defined as the ratio between relative heat absorbed at any stage of gelation and the relative heat input required for complete gelation, suggesting a delay of ca. $10^{\circ} \mathrm{C}$.

Table 2 gathers the cloud point values and, in order to further characterize the thermal rheological response of the polymer solutions, also includes a set of characteristic points. $T_{c 1}$ is defined, for Newtonian regimes, as the temperature corresponding to the viscosity minimum before the steep increase, $T_{c 2}$ represents the temperature at which viscosity sharply decreases for non-Newtonian regimes, and $T_{c 3}$ is the temperature above which $G^{\prime}$ exceeds $G^{\prime \prime} . T_{c 1}$ therefore reflects the sharp increase in viscosity when the gelation process is initiated; $T_{c 2}$ is compatible with a high hydrophobicity of the polymer chains and suggests strong chain aggregation with inhibited intercluster binding; $T_{C 3}$ shows the effect of hydrophobicity in the elastic behavior of the system. The influence of concentration on these characteristic points can also be extracted from the same table. It is seen that the gelation temperature $T_{c 1}$ displays a non-monotonic behavior. The extreme values of $T_{c 1}$ in the concentration range under study differ by $5^{\circ} \mathrm{C}$, which indicates some dependence on temperature. $T_{c 2}$ is, in our findings, almost independent of concentration. It reflects the behavior of the polymer in water that, unperturbed, would lead to gelation. We submit that this point gives also a good assessment of the gelation temperature. $T_{c 3}$ values are observed above $T_{c 1}$ and $T_{c 2}$ and show a similar trend as $T_{c 1}$. This point presents, however, some dependence on the frequency applied in the oscillation test.

The cloud point is lower than $T_{c 1}, T_{c 2}$ or $T_{c 3}$ and has also a negligible dependence on concentration. A similar situation is found for some cellulose systems [29], but it may be also close to [32] or clearly higher than the gelation temperature [30] for others. These discrepancies may be ascribed to different cellulose substituents, but it may also result from different definitions of both cloud point and gelation temperature.

We can schematically summarize the phenomena occurring while heating the polymer solution above $55^{\circ} \mathrm{C}$ as follows: firstly the polymer becomes more hydrophobic, then the solution clouds, and finally the physical gel based on hydrophobic associations is formed.

\subsection{Assessment of active links}

Relaxation times and storage moduli are displayed at different temperatures in Table 1 . These parameters have a direct connec- tion with the previous discussion [33]. While the physical meaning of $G^{\prime}$ is related to the density of the active links in the system, the relaxation time indicates the required time for the solid-liquid transition, which is related to the strength of the active links [34]. Table 1 exhibits three main regimes: at low temperatures $\left(40^{\circ} \mathrm{C}\right)$, there are many weak active links, seen by high $G^{\prime}$ and low relaxation time. This is coherent with the entanglement picture, where many polymer chains touch each other due to overlapping, without any important physical interaction. At such temperatures the polymer is hydrophilic and can easily slide through the polymer matrix according to the reptation picture $[35,36]$. This would lead to small relaxation times.

At intermediate temperatures $\left(60^{\circ} \mathrm{C}\right)$, the active links are in lower amount but are considerably stronger than at lower temperatures. This is compatible with a partial dehydration of the polymer and consequent compaction. The bridging is less effective in this case but the contacts between the polymer chains are more effective.

At higher temperatures $\left(85^{\circ} \mathrm{C}\right)$, above the cloud point, both storage moduli and relaxation times increase. At such temperatures, the polymer-based clusters interact hydrophobically and the strength of the active links is also clearly stronger than the ones at lower temperatures. The polymer association is based on its hydrophobicity and the energy penalty for hydration of the chain inhibits the chain detachment. By comparing the data in Table 1 with Fig. 2b (low shear rates), we can state that the thermal gelation is induced both by an increase of the number of active links, probably related to the increase of the dynamics with temperature, and also by an enhancement of the strength of the polymer associations.

\subsection{Shear rate effects}

Results obtained in this work show the influence of the shear rate in the formation of a gel in HPMC solutions for temperatures above $70^{\circ} \mathrm{C}$. Studies have focused on the concentrations 1 , 2, 5 and $10 \%$, and have shown that for low shear rates there is a steep increase in viscosity when the gelation temperature is attained. These observations are consistent with those obtained in previous work on methyl cellulose solutions using rotational viscometry [13], and agree qualitatively with other previous data [11]. However, for higher shear rates, gel formation is inhibited. Initially the loss of hydration water of the polymer results in a smooth decrease of the viscosity, in a way similar to that observed for Newtonian regime. As the chains become more hydrophobic they tend to form clusters and the high shear rate applied disrupts the intercluster network and prevents gel formation. High shear rates destroy the intercluster bonds and viscosity drops to very low values, even lower than those observed at $25^{\circ} \mathrm{C}$. This behavior has not, to our knowledge, been documented before for HPMC gels.

Some of the above findings may tentatively be explained on the basis of characteristic reorganization times of the system [3]. For low temperatures, the viscosity results from random entanglements (too low values of viscosity for a gel, but too high for a typical liquid solution). The system is thus frequently reorganized, and high shear rates do not substantially disrupt the equilibrium behavior. As the temperature increases, clusters are formed as seen in cloud point studies and, after, gelation sets in. At this point, the system becomes more rigid, and reformation times concomitantly increase. If temperature is further increased, the number of collisions also increases, as does molecular motion, and reformation times decrease. The solid-like behavior of the system now becomes dominant in the measurements, and viscosity increases again as seen for intermediate shear rates (see Fig. 3).

In summary, we have inspected several observables (viscosity profiles at low and high shear rates, optical transmittance of the 


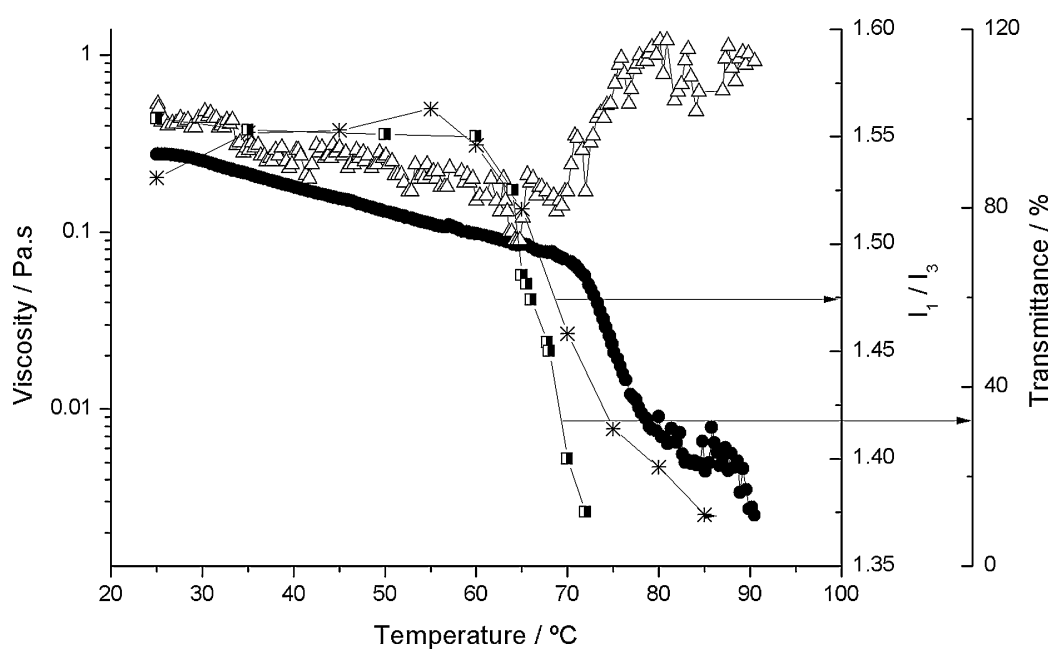

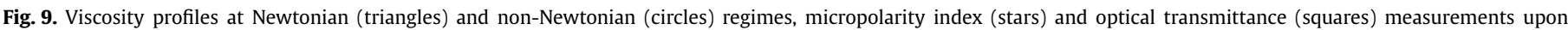
heating for the $1 \%$ HPMC aqueous solution.

solution, micropolarity index) to assess the sequence of processes close to gelation. Additionally, we have looked into PLTM images at different temperatures. All this information is gathered in Fig. 9, in which several of these profiles are superimposed. This picture gives a unified view of the optical properties, viscosity profiles and hydrophobic behavior/aggregation, as previously discussed.

\section{Conclusions}

Results have allowed us to compose a picture of the evolution of HPMC aqueous solutions with temperature. Random entanglements, dominant at room temperature, are still present up to ca. $55^{\circ} \mathrm{C}$. In this range, there is no evidence of association of the polymer in clusters or bundles, and no variation in the micropolarity index. Clouding sets in suddenly and is the first evidence of cluster formation, mainly due to hydrophobic interactions. At even higher temperatures, these clusters are further associated in a three-dimensional network, and gelation can be detected. The use of non-Newtonian shear rates prevents gelation or causes its translation to higher temperatures.

\section{Acknowledgments}

S.M.C.S. and F.E.A. acknowledge Fundação para a Ciência e a Tecnologia, Lisbon (Portugal), for Ph.D. and post-doctoral grants, respectively, references SFRH/BD/30537/2006 and SFRH/BPD/ 26801/2006. We thank Telma Costa for her valuable help with pyrene fluorescence measurements, and Dr. Teresa Roseiro for her important assistance with PLTM studies.

\section{References}

[1] V. Kumar, G.S. Banker, Drug Dev. Ind. Pharm. 19 (1993) 1.

[2] J.F. Kennedy, G.O. Phillips, P.A. Williams, J.L. Picullel, in: Cellulose and Cellulose Derivatives: Physico-Chemical Aspects and Industrial Applications, Cellucon '93 Proceedings, Lund, 1993.

[3] C. Clasen, W.M. Kulicke, Prog. Polym. Sci. 26 (2001) 1839.

[4] J.L. Ford, Int. J. Pharm. 179 (1999) 209.
[5] A. O’Sullivan, Cellulose 4 (1997) 173.

[6] D. Klemm, B. Heublein, H.-P. Fink, A. Bohn, Angew. Chem. Int. Ed. 44 (2005) 3358.

[7] T. Funami, Y. Kataoka, M. Hiroe, I. Asai, R. Takahashi, K. Nishinari, Food Hydrocolloids 21 (2007) 46.

[8] S. Hussain, C. Keary, D.Q.M. Craig, Polymer 43 (2002) 5623.

[9] N. Sarkar, Carbohydr. Polym. 26 (1995) 195.

[10] R. Kita, T. Kaku, K. Kubota, T. Dobashi, Phys. Lett. A 259 (1999) 302.

[11] X.M. Xu, Y.M. Song, Q.N. Ping, Y. Wang, X.Y. Liu, J. Appl. Polym. Sci. 102 (2006) 4066.

[12] C. Sammon, G. Bajwa, P. Timmins, C.D. Melia, Polymer 47 (2006) 577.

[13] N. Sarkar, J. Appl. Polym. Sci. 24 (1979) 1073.

[14] A. Haque, E.R. Morris, Carbohydr. Polym. 22 (1993) 161.

[15] A. Haque, R.K. Richardson, E.R. Morris, M.J. Gidley, D.C. Caswell, Carbohydr. Polym. 22 (1993) 175.

[16] R.N. Ibbett, K. Philp, D.M. Price, Polymer 33 (1992) 4087.

[17] G. Karlström, A. Carlsson, B. Lindman, J. Phys. Chem. 94 (1990) 5005.

[18] M.R. Aguilar, C. Elvira, A. Gallardo, B. Vásquez, J.S. Róman, in: N. Ashammakhi, R. Reis, E. Chiellini (Eds.), in: Topics in Tissue Engineering, vol. 3, 2007, p. 1.

[19] Y. Sekiguchi, C. Sawatari, T. Kondo, Carbohydr. Polym. 53 (2003) 145.

[20] J.D. Ferry, Viscoelastic Properties of Polymers, Wiley, New York, 1980.

[21] K. Holmberg, B. Jönsson, B. Kronberg, B. Lindman, Surfactants and Polymers in Aqueous Solution, Wiley, Chichester, 2003.

[22] M. Egermayer, M. Karlberg, L. Piculell, Langmuir 20 (2004) 2208.

[23] P. Zheng, L. Li, X. Hu, X. Zhao, J. Polym. Sci. B 42 (2004) 1849.

[24] Y. Xu, L. Li, Polymer 46 (2005) 7410.

[25] A. Ridell, H. Evertsson, S. Nilsson, J. Colloid Interface Sci. 247 (2002) 381.

[26] D. Ostrovskii, A.L. Kjoniksen, B. Nystrom, L.M. Torell, Macromolecules 32 (1999) 1534.

[27] J. Desbrières, M. Hirrien, S.B. Ross-Murphy, Polymer 41 (2000) 2451.

[28] L. Li, P.M. Thangamathesvaran, C.Y. Yue, K.C. Tam, X. Hu, Y.C. Lam, Langmuir 17 (2001) 8062.

[29] J. Desbrieres, M. Hirrien, M. Rinaudo, Carbohydr. Polym. 37 (1998) 145.

[30] M. Hirrien, C. Chevillard, J. Desbrieres, M.A.V. Axelos, M. Rinaudo, Polymer 39 (1998) 6251.

[31] S.C. Joshi, Y.C. Lam, B.K. Tan, S.Q. Liu, in: Int. Conf. on Biomedical and Pharmaceutical Engineering, 2006, 545 pp.

[32] K. Kobayashi, C. Huang, T.P. Lodge, Macromolecules 32 (1999) 7070.

[33] F.E. Antunes, R.O. Brito, E.F. Marques, B. Lindman, M. Miguel, J. Phys. Chem. B 111 (2007) 116.

[34] H.A. Barnes, J.F. Hutton, K. Walters, An Introduction to Rheology, Elsevier, Amsterdam, 1989.

[35] P.G. De Gennes, J. Chem. Phys. 55 (1971) 572.

[36] F. Tanaka, Macromolecules 22 (1989) 1988. 